\title{
ON THE COEFFICIENTS OF AN ASYMPTOTIC EXPANSION OF SPHERICAL FUNCTIONS ON SYMMETRIC SPACES
}

\author{
JOHN J. H. MILLER AND D. J. SIMMS
}

ABSTRACT. The asymptotic expansion of a spherical function on a symmetric space of noncompact type, obtained by HarishChandra, is a finite linear combination of expansions of the form $\Phi_{\theta}=\sum_{\mu}{ }^{\prime} \Gamma_{\mu}(\theta) e^{\theta-\mu}$. In this paper it is proved that $\lim _{t \rightarrow \infty} \Gamma_{\mu}(t e-\bar{\rho})$ is finite and rational for any $e$, where $\bar{\rho}$ is the restriction of half the sum of the positive roots.

1. Introduction. Let $G$ be a connected real semisimple Lie group with finite centre and $K$ a maximal compact subgroup. Let $G=K A N$ be an Iwasawa decomposition of $G$, relative to a system $\{\alpha\}$ of simple roots of $G$, and among their restrictions $\{\bar{\alpha}\}$ to the Lie algebra $\mathfrak{A}$ of $A$ choose a system $\bar{\alpha}_{1}, \cdots, \bar{\alpha}_{l}$ of simple restricted roots of the symmetric space $G / K$. Denote by $L$ the lattice of all integral combinations $\mu=\sum_{i=1}^{l} m_{i} \bar{\alpha}_{i}$ and let $L^{+}$be the set of $\mu$ with each $m_{i}$ nonnegative. Write $\nu \leqq \mu$ if $\mu-\nu \in L^{+}$. Regard each $\mu$ in the complex dual $\Lambda^{C}$ of $\mathfrak{A}$ as a linear form on $\Lambda^{C}$ via the Killing form $\langle\cdot, \cdot\rangle$. Let $\Delta_{+}$be the set of positive roots of $G, \bar{\rho}$ the restriction to $\mathfrak{A}$ of half the sum of the positive roots; and $Q=\prod_{\beta \in P_{+}}\left(e^{\beta}-e^{-\beta}\right)$ where $P_{+}=\left\{\beta \in \Delta_{+} \mid \bar{\beta} \neq 0\right\}$.

For each $\mu \in L$ let ' $\Gamma_{\mu}$ be the rational function on $\Lambda C$ defined by the recursion relation

$$
\begin{aligned}
' \Gamma_{\mu} & =0 \quad \text { if } \mu \in L-L^{+}, \\
' \Gamma_{0} & =1, \\
c_{\mu}{ }^{\prime} \Gamma_{\mu} & =\sum_{\beta \in \mathcal{J}_{+}} \sum_{k=1}^{\infty} \gamma(\beta)_{\mu-2 k \beta} \Gamma^{\prime}{ }_{\mu-2 k \beta} \quad \text { if } \mu \in L^{+}-\{0\},
\end{aligned}
$$

where the coefficients are the functions on $\Lambda^{C}$ given by

$$
c_{\mu}=\langle\mu, \mu-2 \bar{\rho}\rangle-2 \mu, \quad \gamma(\beta)_{\mu}=2\langle\mu, \bar{\beta}\rangle-2 \bar{\beta} .
$$

Received by the editors March 9, 1972.

AMS (MOS) subject classifications (1970). Primary 43A90; Secondary 17B20, 22E30, 22E60.

Key words and phrases. Symmetric space of noncompact type, spherical function, coefficient of asymptotic expansion, radial limit.

c) American Mathematical Society 1973 
According to Harish-Chandra [3], each spherical function on $G / K$, when transferred to $\mathfrak{A}$ by the exponential, can be written as a finite linear combination of asymptotic expansions of the form

$$
\Phi_{\theta}=\sum_{\mu \in L} \Gamma_{\mu}(\theta) e^{\theta-\mu}
$$

In [7] we showed that for $e \in \Lambda$ the radial limit $\lim _{t \rightarrow \infty} ' \Gamma_{\mu}($ ite $-\bar{\rho})$ is finite and independent of $e$ except possibly for those exceptional directions of $e$ for which $\langle e, v\rangle=0$ for some $0 \leqq v \leqq \mu$. In this paper we show, by a different method, that the radial $\operatorname{limit}_{\lim _{t \rightarrow \infty}}{ } \Gamma_{\mu}(t e-\bar{\rho})$ is finite for all $e \in \Lambda^{C}$ without exception. We also calculate ' $\Gamma_{\mu}(\theta)$ explicitly when $\mu$ is a multiple of a simple restricted root which is not itself a multiple of any other positive restricted root. This shows in particular that the radial limit in an exceptional direction may differ from the limit in a nonexceptional direction.

\section{The radial limits.}

THEOREM. Let $e, a \in \Lambda^{C}, \mu \in L^{+}$and suppose that $\langle e, v\rangle \neq 0$ for all $0 \leqq v \leqq \mu$. Then $\lim _{t \rightarrow \infty ; t \in C} \backslash \Gamma_{\mu}(t e+a)$ exists and is equal to the positive rational number $A_{\mu}$ defined by

$$
e^{\bar{\rho}} Q^{-1 / 2}=\prod_{\beta \in P_{+}}\left(1-e^{-2 \beta}\right)^{-1 / 2}=\sum_{\mu \in L} A_{\mu} e^{-\mu} .
$$

For all $e \in \Lambda^{C}$, without exception, $\lim _{t \rightarrow \infty ; t \in C} \Gamma_{\mu}(t e-\bar{\rho})$ exists and is a rational number.

Proof. Let the rational functions $C_{\mu}$ and the rational numbers $D_{\mu}$ be defined by

$$
Q^{1 / 2} \Phi_{\theta}=e^{\theta+\bar{\rho}} \sum_{\mu \in L} C_{\mu}(\theta) e^{-\mu}, \quad Q^{-1 / 2} \nabla^{2}\left(Q^{1 / 2}\right)=\sum_{\mu \in L} D_{\mu} e^{-\mu}
$$

where $\nabla^{2}$ is the Laplacian on $\mathfrak{U}$. We note that $A_{\mu}, C_{\mu}$ and $D_{\mu}$ are all zero for $\mu \in L-L^{+}, A_{0}=1, C_{0} \equiv 1, D_{0}=\langle\bar{\rho}, \bar{\rho}\rangle$, and $A_{\mu} \geqq 0$ for all $\mu \in L$. By a result of Gangolli [2] in a form given by Helgason [5, Chapter II, $\$ 2$ p. 38] we have:

$$
\{\langle\mu, \mu\rangle-2\langle\mu, \theta+\bar{\rho}\rangle\} C_{\mu}(\theta)=\sum_{v \in L ; v \neq \mu} D_{\mu-\nu} C_{v}(\theta) .
$$

Now fix $e, a \in \Lambda^{C}$ and put $\theta=t e+a, t \in C$, to get

$$
\left\{\langle\mu, \mu-2 a-2 \bar{\rho}\rangle-2 t^{\prime}\langle\mu, e\rangle\right\} C_{\mu}(t e+a)=\sum_{v \in L: v \neq \mu} D_{\mu-\nu} C_{v}(t e+a) .
$$

In particular, for $a=-\bar{\rho}$, we have

$$
\{\langle\mu, \mu\rangle-2 t\langle\mu, e\rangle\} C_{\mu}(t e-\bar{\rho})=\sum_{v \in L: v \neq \mu} D_{\mu-\nu} C_{v}(t e-\bar{\rho}) .
$$


We now distinguish two cases, depending on $a$ and on the direction of $e$.

Case (i). For all $a, e \in \Lambda^{c}$ such that $\langle e, v\rangle \neq 0$ for all $0 \leqq \nu \leqq \mu$ ('nonexceptional directions of $e$ ): In this case we let $t \rightarrow \infty$ in (2.1) and use induction on $\mu$ to conclude that

$$
\lim _{t \rightarrow \infty} C_{\mu}(t e+a)=0
$$

for all $\mu \in L^{+}, \mu \neq 0$.

Case (ii). For $a=-\bar{\rho}$ and for all $e \in \Lambda^{C}$ : In this case we let $t \rightarrow \infty$ in (2.2) and use induction on $\mu$ to conclude that

$$
\lim _{t \rightarrow \infty} C_{\mu}(t e-\bar{\rho})
$$

is finite and rational for all $\mu \in L$. Now

$$
\sum_{\mu \in L} \cdot \Gamma_{\mu}(\theta) e^{\theta-\mu}=\Phi_{\theta}=\left(\sum_{\mu \in L} A_{\mu} e^{-\mu}\right) \cdot\left(\sum_{\mu \in L} C_{\mu}(\theta) e^{\theta-\mu}\right)
$$

therefore

$$
{ }^{\prime} \Gamma_{\mu}(\theta)=\sum_{v \in L} A_{\mu-v} C_{v}(\theta) .
$$

For Case (i), $\langle e, v\rangle \neq 0$ for all $0 \leqq \nu \leqq \mu$, so that

$$
\lim _{t \rightarrow \infty} \Gamma_{\mu}(t e+a)=\sum_{v \in L} A_{\mu-v} \lim _{t \rightarrow \infty} C_{v}(t e+a)=A_{\mu}
$$

by (2.3). Also, Case (ii), we have for all $e \in \Lambda^{c}$

$$
\lim _{t \rightarrow \infty} \Gamma_{\mu}(t e-\bar{\rho})=\sum_{v \in L} A_{\mu-v} \lim _{t \rightarrow \infty} C_{v}(t e-\bar{\rho})
$$

which is finite and rational by (2.4). This completes the proof.

REMARK. The Theorem shows that the rational numbers $A_{\mu}$ are the same as those introduced in [7] by the recursion relation

$$
\mu A_{\mu}=\sum_{\beta \in P_{+}} \sum_{k=1}^{\infty} \bar{\beta} A_{\mu-2 k \beta} .
$$

This recursion relation can easily be obtained by applying the gradient operator $\nabla$ to $e^{\ngtr} Q^{-1 / 2}$ to get

$$
\begin{aligned}
\sum_{\mu \in L} \mu A_{\mu} e^{-\mu} & =\sum_{\beta \in P_{+}} \bar{\beta} e^{-2 \beta}\left(1-e^{-2 \beta}\right)^{-1} \prod_{\beta \in P_{+}}\left(1-e^{-2 \beta}\right)^{-1 / 2} \\
& =\sum_{\beta_{\in} I_{+}+} \bar{\beta} \sum_{k=1}^{\infty} e^{-2 k \beta} \sum_{\mu \in L} A_{\mu} e^{-\mu}
\end{aligned}
$$

Equating the coefficients of $e^{-\mu}$ gives (2.5). 
3. Some calculations and estimates for ' $\Gamma_{\mu}$ and $A_{\mu}$. The following proposition deals with the special case $q=0$ of $[4, \S 5$, p. 304]. It gives an improvement of Helgason's estimate for this case.

PROPOSITION. Let $\alpha$ be a root of $G$ such that $\bar{\alpha}$ is one of the restricted simple roots $\bar{\alpha}_{1}, \cdots, \bar{\alpha}_{l}$. Let $p$ be the number of positive roots of $G$ whose restriction to $\mathfrak{A}$ is equal to $\bar{\alpha}$, and suppose that $\bar{\alpha}$ is not a scalar multiple of any other restricted root. Let $\eta=\langle\bar{\alpha}, \bar{\alpha}\rangle^{-1} \bar{\alpha}$. Then for all $m \geqq 1, \theta \in \Lambda^{c}$ we have $\eta(\bar{\rho})=\frac{1}{2} p$ and

$$
\Gamma_{2 m \bar{\alpha}}(\theta)=\prod_{r=1}^{m}\left(1+\frac{p-2}{2 r}\right)\left(1+\frac{p-2}{2 r-p-2 \eta(\theta)}\right) .
$$

Proof. Put $\mu$ equal to $2 m \bar{\alpha}$ and $2(m-1) \bar{\alpha}$ in turn in (1.1) to get

$$
\begin{gathered}
c_{2 m \bar{\alpha}} \Gamma_{2 m \bar{\alpha}}=\sum_{k=1}^{\infty} p \gamma(\alpha)_{2(m-k) \bar{\alpha}} \Gamma_{2(m-k) \bar{\alpha}}, \\
c_{2(m-1) \bar{\alpha}} \Gamma_{2(m-1) \bar{\alpha}}=\sum_{k=1}^{\infty} p \gamma(\alpha)_{2(m-k-1) \bar{\alpha}} \Gamma_{2(m-k-1) \bar{\alpha}} .
\end{gathered}
$$

Subtracting we get

$$
c_{2 m \bar{\alpha}} \Gamma_{2 m \bar{\alpha}}-c_{2(m-1) \bar{\alpha}} \Gamma_{2(m-1) \bar{\alpha}}=p \gamma(\alpha)_{2(m-1) \bar{\alpha}} \Gamma_{2(m-1) \bar{\alpha}} .
$$

It is an easy and well-known consequence of $[1, \mathrm{VI}, \S 1.6$, Corollary 1$]$ that $\langle\bar{\alpha}, \bar{\rho}\rangle=\frac{1}{2} p\langle\bar{\alpha}, \bar{\alpha}\rangle$. Thus $\eta(\bar{\rho})=\frac{1}{2} p$. From (1.2) we have

$$
\begin{aligned}
& c_{2 m \bar{\alpha}}(\theta)=\langle 2 m \bar{\alpha}, 2 m \bar{\alpha}-2 \bar{\rho}-2 \theta\rangle=4 m[m-\eta(\bar{\rho}+\theta)]\langle\bar{\alpha}, \bar{\alpha}\rangle, \\
& \gamma_{2 m \bar{\alpha}}(\theta)=\langle 4 m \bar{\alpha}, \bar{\alpha}\rangle-\langle 2 \bar{\alpha}, \theta\rangle=[4 m-2 \eta(\theta)]\langle\bar{\alpha}, \bar{\alpha}\rangle .
\end{aligned}
$$

Substitution in (3.2) gives, with the use of $\eta(\bar{\rho})=\frac{1}{2} p$,

$$
\begin{aligned}
\Gamma_{2 m \bar{\alpha}}(\theta) & =\frac{[4(m-1)+2 p][m-1-\eta(\theta)]}{4 m\left[m-\frac{1}{2} p-\eta(\theta)\right]} \cdot \Gamma_{2(m-1) \bar{\alpha}}(\theta) \\
& =(1+(p-2) / 2 m)(1+(p-2) /(2 m-p-2 \eta(\theta))) \Gamma^{\prime} \Gamma_{2(m-1) \bar{\alpha}}(\theta) .
\end{aligned}
$$

This gives (3.1).

COROllaries. Let $m \geqq 1$.

(i) Since $\eta(\bar{\rho})=\frac{1}{2} p$ we have, for all $\lambda \in \Lambda^{C}$,

$$
' \Gamma_{2 m \bar{\alpha}}(\lambda-\bar{\rho})=\prod_{r=1}^{m}\left(1+\frac{p-2}{2 r}\right)\left(1+\frac{p-2}{2 r-2 \eta(\lambda)}\right) .
$$

(ii) In a 'nonexceptional' direction of $e \in \Lambda^{C}$ given by $\langle\bar{\alpha}, e\rangle \neq 0$ we have 
$\eta(e) \neq 0$ and therefore

$$
\begin{aligned}
A_{2 m \bar{\alpha}} & =\lim _{t \rightarrow \infty} \Gamma_{2 m \bar{x}}(t e-\bar{\rho}) \\
& =\lim _{t \rightarrow \infty} \prod_{r=1}^{m}\left(1+\frac{p-2}{2 r}\right)\left(1+\frac{p-2}{2 r-2 t \eta(e)}\right)=\prod_{r=1}^{m}\left(1+\frac{p-2}{2 r}\right) .
\end{aligned}
$$

(iii) In an 'exceptional direction' given by $\langle\bar{\alpha}, \lambda\rangle=0$ we have $\eta(\lambda)=0$ and therefore by (i) and (ii)

$$
\Gamma_{2 m \bar{\alpha}}(\lambda-\bar{\rho})=\prod_{r=1}^{m}\left(1+\frac{p-2}{2 r}\right)^{2}=A_{2 m \bar{\alpha}}^{2} .
$$

(iv) If $p=1$ then for $\lambda \in \Lambda$ we have

$$
\left|\Gamma_{2 m \bar{\alpha}}(i \lambda-\bar{\rho})\right|=\left|\prod_{r=1}^{m}\left(1-\frac{1}{2 r}\right)\left(1-\frac{1}{2 r-2 i \eta(\lambda)}\right)\right|<\prod_{r=1}^{m}\left(1-\frac{1}{2 r}\right)
$$

since $i \eta(\lambda)$ is pure imaginary.

(v) If $p=2$ then ' $\Gamma_{2 m \bar{\alpha}}(\theta)=1$ for all $\theta \in \Lambda^{C}$.

(vi) If $p>2$ and $\lambda \in \Lambda$, then the absolute value of $1+(p-2) /(2 r-2 i \eta(\lambda))$ is greater than 1 and less than or equal to $1+(p-2) / 2 r$. Therefore, using (ii) we have $A_{2 m \bar{\alpha}}<\left|\Gamma_{2 m \bar{\alpha}}(i \lambda-\bar{\rho})\right| \leqq A_{2 m \bar{\alpha}}^{2}$.

Remark. The conditions of the Proposition are satisfied provided that $\alpha$ is represented by a node of the form $\bigcirc$, and not of the form $\odot$ in Tables 4 and 8 on pp. 119 and 146 of [6, Chapter VII].

ACKNOWLEDGEMENT. We should like to thank Sigurdur Helgason for stimulating discussions and for making available to us the manuscript of [5] before publication.

\section{REFERENCES}

1. N. Bourbaki, Éléments de mathématiques. XXVI. Groupes et algèbres de Lie, Actualités Sci. Indust., no. 1285, Hermann, Paris, 1960. MR 24 \#A2641.

2. R. Gangolli, On the Plancherel formula and the Paley-Wiener theorem for spherical functions on semisimple Lie groups, Ann. of Math. (2) 93 (1971), 150-165.

3. Harish-Chandra, Spherical functions on a semisimple Lie group. I, Amer. J. Math. 80 (1958), 241-310. MR 20 \#925.

4. S. Helgason, An analogue of the Paley-Wiener theorem for the Fourier transform on certain symmetric spaces, Math. Ann. 165 (1966), 297-308. MR 36 \#6545.

5. - A duality for symmetric spaces with applications to group representations, Advances in Math. 5 (1970), 1-154. MR 41 \#8587.

6. O. Loos, Symmetric spaces. II: Compact spaces and classification, Benjamin, New York, 1969. MR 39 \#365b.

7. John J. H. Miller and D. J. Simms, Radial limits of the rational functions ' $\Gamma_{\mu}$ of Harish-Chandra, Proc. Roy. Irish Acad. Sect. A 68 (1969), 41-47. MR 40 \#7391.

School of Mathematics, Trinity College, Dublin, Ireland

Current address (D. J. Simms): Mathematisches Institut der Universität Bonn, 53 Bonn, Wegelerstrasse 10, Germany 